\title{
Propagation of Rayleigh Surface Wave in a Two-Temperature Thermoelastic Solid Half-Space with Diffusion
}

\author{
Sangeeta Kumari ${ }^{{ }^{*}}$ and Baljeet Singh $^{2}$ \\ ${ }^{1}$ Department of Mathematics, Chandigarh University, Gharuan, Punjab, India \\ ${ }^{2}$ Department of Mathematics, Post Graduate Government College, Sector-11, Chandigarh - 160 011, India \\ Email: sangwan.sangeeta@gmail.com
}

\begin{abstract}
Lord and Shulman theory of generalized thermoelasticity is applied to formulate the field equations governing a two-temperature thermoelastic medium with diffusion. The equations are reduced for an isotropic case in $\mathrm{x}-\mathrm{z}$ plane. These equations are solved for general surface wave solutions. Particular solutions satisfying the required radiations conditions are obtained in a half-space. Applying suitable boundary conditions at free surface, a secular equation for wave speed of Rayleigh surface wave is obtained. The wave speed of Rayleigh wave is computed by using Fortran Program of iteration method for a particular example of the half-space. The effects of two-temperature parameter, frequency, thermodiffusion parameter and diffusion relaxation time are observed graphically on the wave speed of Rayleigh wave.
\end{abstract}

Keywords: Generalized thermoelasticity; Rayleigh wave; two-temperature; diffusion; wave speed.

\section{Nomenclature}

$\rho$ is the density of the medium.

$\lambda, \mu$ are the Lame's constant.

$\alpha_{t}$ is the coefficient of linear thermal expansion.

$\alpha_{c}$ is the coefficient of diffusion expansion.

$T$ is the absolute temperature.

$T_{0}$ is the temperature of the medium in its natural state.

$\Phi$ is conductive temperature.

$\sigma_{i j}$ are the components of stress tensor.

$e_{i j}$ are the components of strain tensor.

$u_{i}$ are the components of displacement vector.

$S$ is the entropy per unit mass.

$P$ is the chemical potential per unit mass.

$C$ is the mass concentration.

$c_{E}$ is the specific heat at constant strain.

$K$ is the coefficient of thermal conductivity.

$D^{*}$ is the thermo-diffusion constant.

$\tau_{0}$ is the thermal relaxation time.

$\tau$ is the diffusion relaxation time.

$a^{*}>0$ is a two temperature parameter.

$a, b$ are constants to measure thermo-diffusion effects and diffusive effects.

\section{Introduction}

Lord and Shulman [10] and Green and Lindsay [6] formulated theories of generalized thermoelasticity which remove the shortcomings of classical theory of thermoelasticity developed by Biot [1]. Dhaliwal and Sherief [5] extended the Lord and Shulman theory for anisotropic case. Hetnarski and Ignaczak [8] reviewed these theories of generalized thermoelasticity in detail. 
The diffusion phenomenon in solids is of great importance due to its applications in geophysics and industries. This phenomenon is used for extraction oil more efficiently from oil deposits. The coupling of temperature, mass diffusion and strain fields is known as thermodiffusion in an elastic solid. Nowacki $[11,12]$ developed the coupled theory of thermoelastic diffusion in solids. Sherief et al. [14] developed the generalized theory of thermoelastic diffusion, which permits finite speed of thermal signals. Later, Singh $[15,16]$ showed the existence of three coupled longitudinal waves and a shear wave in a two-dimensional model of generalized thermoelastic solid with diffusion. He also studied the reflection of P and SV waves from free surface of a half-space. Thereafter, many researchers studied various problems on wave propagation in generalized thermoelasticity with diffusion.

The development of a two-temperature theory of thermoelasticity is due to Gurtin and Williams [7], Chen and Gurtin [2] and Chen et al. [3, 4]. Warren and Chen [18] and Puri and Jordan [13] studied the propagation of plane waves in two-temperature themoelasticity. Later in 2006, Youssef [19] developed the theory of two-temperature generalized thermoelasticity. Kumar and Mukhopadhay [9] studied the effects of thermal relaxations on wave propagation in theory of two-temperature thermoelasticity. Singh [17] studied the Rayleigh surface wave at a stress free thermally insulated surface of an isotropic, linear, and homogeneous two-temperature thermoelastic solid half-space in the context of Lord and Shulman theory of generalized thermoelasticity.

In this paper the propagation of Rayleigh surface wave in a two-temperature thermoelastic solid with diffusion is studied. The secular equation for wave speed of Rayleigh wave is obtained and solved numerically to observe the effects of thermo-diffusion parameter, diffusion relxation time, frequency and two-temperature parameter on the wave speed of Rayleigh wave.

\section{Basic Equations}

Following Sherief et al [14], the governing equations for a linear, isotropic and homogeneous elastic solid with generalized thermodiffusion at constant temperature $\mathrm{T}$ in the absence of body force are:

(i) The displacement-strain relation

$$
e_{i j}=\frac{1}{2}\left(u_{i, j}+u_{j, i}\right)
$$

(ii) The energy equation

$$
-q_{i, i}=\rho T_{0} \dot{S}
$$

(iii) The modified Fourier's law

$$
-K_{i j} \Phi_{, j}=q_{i}+\tau_{0} \dot{q}_{i}
$$

(iv) The equation of motion

$$
\mu u_{i, j j}+(\lambda+\mu) u_{j, i j}-\beta_{1} \Theta_{, i}-\beta_{2} C_{, i}=\rho \ddot{u}_{i},
$$

(v) The equation of heat conduction:

$$
\rho c_{E}\left(\dot{\Theta}+\tau_{0} \ddot{\Theta}\right)+\beta_{1} T_{0}\left(\dot{e}+\tau_{0} \ddot{e}\right)+a T_{0}\left(\dot{C}+\tau_{0} \ddot{C}\right)=K \Phi_{, i i},
$$

(vii) The equation of mass diffusion:

$$
D^{*} \beta_{2} e_{, i i}+D^{*} a \Theta_{, i i}+\dot{C}+\tau \ddot{C}-D^{*} b C_{, i i}=0,
$$

(viii)Two temperature relation

$$
\Phi-\Theta=a^{*} \Phi_{, i i}
$$

(ix)The constitutive equations

$$
\begin{gathered}
\sigma_{i j}=2 \mu e_{i j}+\delta_{i j}\left(\lambda e_{k k}-\beta_{1} \Theta-\beta_{2} C\right), \\
\rho T_{0} S=\rho c_{E} \Theta+\beta_{1} T_{0} e_{k k}+a T_{0} C, \\
P=-\beta_{2} e_{k k}+b C-a \Theta,
\end{gathered}
$$

where $\beta_{1}=(3 \lambda+2 \mu) \alpha_{t}$ and $\beta_{2}=(3 \lambda+2 \mu) \alpha_{c} . \Theta=T-T_{0}$. The dots over variables denote the derivatives with respect to time. 


\section{Formulation of Problem and Solution}

We consider a linear, homogeneous and isotropic two-temperature thermoelastic medium with diffusion of an infinite extent with Cartesian coordinates system $(x, y, z)$. The origin is taken on the plane surface and $z-$ axis is taken normally into the medium $(z \geq 0)$. The surface $z=0$ is assumed stress free and thermally insulated. The present study is restricted to $x-z$ plane, with the displacement vector $\boldsymbol{u}=\left(u_{1}, 0, u_{3}\right)$, where

$$
u_{1}=\frac{\partial \phi}{\partial x}-\frac{\partial \psi}{\partial z}, u_{3}=\frac{\partial \phi}{\partial z}+\frac{\partial \psi}{\partial x}
$$

With the help of equations (7)and (11), the equations (4) to (6) reduce to

$$
\begin{gathered}
\mu\left(\psi_{, 11}+\psi_{, 33}\right)=\rho \ddot{\psi}, \\
(\lambda+2 \mu)\left(\phi_{, 11}+\phi_{, 33}\right)-\beta_{1} \Theta-\beta_{2} C=\rho \ddot{\phi}, \\
K\left(\Phi_{, 11}+\Phi_{, 33}\right)=\rho c_{E} \tau_{m} \dot{\Theta}+\beta_{1} T_{0} \tau_{m} \frac{\partial}{\partial t} \nabla^{2} \phi+a T_{0} \tau_{m} \dot{C}, \\
D^{*} \beta_{2} \nabla^{2}\left(\phi_{, 11}+\phi_{, 33}\right)+D^{*} a\left(\Theta_{, 11}+\Theta_{, 33}\right)-D^{*} b\left(C_{, 11}+C_{, 33}\right)+\tau_{n} \dot{C}=0
\end{gathered}
$$

where $\tau_{m}=1+\tau_{0} \frac{\partial}{\partial t}$ and $\tau_{n}=1+\tau \frac{\partial}{\partial t}$.

For propagation of thermoelstic Rayleigh surface waves along x-axis, the potential functions $\phi, \Phi, C$, are taken in the following form:

$$
[\phi, \Phi, C, \psi]=[\phi \hat{(z)}, \Phi \hat{(z)}, C(z) \hat{, \psi} \hat{(z)}] e^{\iota(\eta x-\chi t)}
$$

Substituting $\psi$ from equation (16) in equation (12), we obtain

$$
\frac{m_{4}^{2}}{\eta^{2}}=1-\frac{c^{2}}{c_{2}^{2}}
$$

where $c_{2}^{2}=\mu / \rho$.

Substituting $\phi, \Phi, C$ from equation (16) in equations (13) to (15), we obtain a homogeneous system of three equations in $\phi, \Phi$ and $C$ and the non-trivial solution of which requires

$$
D^{6}-L D^{4}+M D^{2}-N=0
$$

where $L, M, N$ are given in Appendix I.

Therefore, the general solutions of equations (12) to (15) are written as

$$
\begin{gathered}
(z)=\left[P \exp \left(-m_{4} z\right)+P^{\prime} \exp \left(m_{4} z\right)\right] e^{\iota(\eta x-\chi t)}, \\
\phi(z)=\sum_{i=1}^{3}\left[Q_{i} \exp \left(-m_{i} z\right)+Q_{i}{ }^{\prime} \exp \left(m_{i} z\right)\right] e^{\iota(\eta x-\chi t)}, \\
\Phi(z)=\sum_{i=1}^{3}\left[R_{i} \exp \left(-m_{i} z\right)+R_{i}{ }^{\prime} \exp \left(m_{i} z\right)\right] e^{\iota(\eta x-\chi t)}, \\
C(z)=\sum_{i=1}^{3}\left[S_{i} \exp \left(-m_{i} z\right)+{S^{\prime}}^{\prime} \exp \left(m_{i} z\right)\right] e^{\iota(\eta x-\chi t)},
\end{gathered}
$$

where $P, Q_{i}, R_{i}, S_{i}, P^{\prime}, Q_{i}{ }^{\prime}, R_{i}{ }^{\prime}, S_{i}{ }^{\prime}$ are the arbitrary constants. Here $R_{i}=F_{i} Q_{i}, S_{i}=F_{i}{ }^{*} Q_{i}$ and the expressions for $F_{i}$ and $F_{i}{ }^{*},(i=1,2,3)$ are given in the Appendix I. $m_{i}{ }^{2}$ are the roots of the equation

$$
x^{3}-L x^{2}+M x-N=0 .
$$


In general, the roots $m_{i},(i=1,2,3)$ are complex, therefore, we assume $\operatorname{Re}\left(m_{i}\right)>0$ for surface wave solutions. With the following radiation conditions

$$
(z), \phi(z), \Phi(z), C(z) \rightarrow 0 \text { as } z \rightarrow \infty,
$$

the solutions (19) to (22) reduce to particular solutions in the half-space $z>0$.

$$
\begin{gathered}
(z)=P \exp \left(-m_{4} z\right) e^{\iota(\eta x-\chi t)}, \\
\phi(z)=\sum_{i=1}^{3} Q_{i} \exp \left(-m_{i} z\right) e^{\iota(\eta x-\chi t)}, \\
\Phi(z)=\sum_{i=1}^{3} F_{i} Q_{i} \exp \left(-m_{i} z\right) e^{\iota(\eta x-\chi t)}, \\
C(z)=\sum_{i=1}^{3} F_{i}^{*} Q_{i} \exp \left(-m_{i} z\right) e^{\iota(\eta x-\chi t)}
\end{gathered}
$$

\section{Derivation of Secular Equation}

The appropriate boundary conditions at stress free surface $z=0$ are

$$
\sigma_{z z}=0, \quad \sigma_{z x}=0, \quad \frac{\partial P}{\partial z}=0, \quad \frac{\partial \Phi}{\partial z}=0 .
$$

Making use of the solutions (25) to (28) in boundary conditions (29), we obtain a homogeneous system of four equations in $P, Q_{1}, Q_{2}$ and $Q_{3}$, which has non trivial solution if

$$
\begin{aligned}
& 4 \mu \frac{m_{4}}{\eta}\left[\frac{m_{1}}{\eta}\left(Y_{2} \frac{m_{3}}{\eta} \frac{F_{3}}{\eta^{2}}-Y_{3} \frac{m_{2}}{\eta} \frac{F_{2}}{\eta^{2}}\right)-\frac{m_{2}}{\eta}\left(Y_{1} \frac{m_{3}}{\eta} \frac{F_{3}}{\eta^{2}}-Y_{3} \frac{m_{1}}{\eta} \frac{F_{1}}{\eta^{2}}\right)+\frac{m_{3}}{\eta}\left(Y_{1} \frac{m_{2}}{\eta} \frac{F_{2}}{\eta^{2}}-Y_{2} \frac{m_{1}}{\eta} \frac{F_{1}}{\eta^{2}}\right)\right] \\
& -\left(\frac{m_{4}^{2}}{\eta^{2}}+1\right)\left[X_{1}\left(Y_{2} \frac{m_{3}}{\eta} \frac{F_{3}}{\eta^{2}}-Y_{3} \frac{m_{2}}{\eta} \frac{F_{2}}{\eta^{2}}\right)-X_{2}\left(Y_{1} \frac{m_{3}}{\eta} \frac{F_{3}}{\eta^{2}}-Y_{3} \frac{m_{1}}{\eta} \frac{F_{1}}{\eta^{2}}\right)+X_{3}\left(Y_{1} \frac{m_{2}}{\eta} \frac{F_{2}}{\eta^{2}}-Y_{2} \frac{m_{1}}{\eta} \frac{F_{1}}{\eta^{2}}\right)\right]=0
\end{aligned}
$$

where

$X_{i}=-\lambda+(\lambda+2 \mu) \frac{m_{i}^{2}}{\eta^{2}}-\beta_{1}\left[1-a^{*} \eta^{2}\left(-1+\frac{m_{i}^{2}}{\eta}\right)\right] \frac{F_{i}}{\eta^{2}}-\beta_{2} \frac{F_{i}^{*}}{\eta^{2}},(i=1,2,3)$

$Y_{i}=\beta_{2}\left(-1+\frac{m_{i}^{2}}{\eta^{2}}\right)+b \frac{F_{i}^{*}}{\eta^{2}}-a\left[1-a^{*} \eta^{2}\left(-1+\frac{m_{i}{ }^{2}}{\eta}\right)\right] \frac{F_{i}}{\eta^{2}}$.

The equation (30) is the secular equation for wave speed of Rayleigh wave at free surface of a twotemperature thermoelastic solid half-space with diffusion.

\section{Particular Cases}

(i) In absence of diffusion parameters, the equation (30)reduces to

$$
X_{1}\left[\frac{m_{2}}{\eta} \frac{m_{3}}{\eta} \frac{F_{3}}{\eta^{2}}-\frac{m_{3}}{\eta} \frac{m_{2}}{\eta} \frac{F_{2}}{\eta^{2}}\right]-X_{2}\left[\frac{m_{1}}{\eta} \frac{m_{3}}{\eta} \frac{F_{3}}{\eta^{2}}-\frac{m_{3}}{\eta} \frac{m_{1}}{\eta} \frac{F_{1}}{\eta^{2}}\right]+X_{3}\left[\frac{m_{1}}{\eta} \frac{m_{2}}{\eta} \frac{F_{2}}{\eta^{2}}-\frac{m_{2}}{\eta} \frac{m_{1}}{\eta} \frac{F_{1}}{\eta^{2}}\right]=0,
$$

where the expressions for $X_{i}, Y_{i}, \frac{m_{i}}{\eta}, \frac{F_{i}}{\eta^{2}}$ and $\frac{F_{i}{ }^{*}}{\eta^{2}}$ reduce accordingly.

(ii)In absence of two-temperature parameter, the equation (30) reduces to

$$
\begin{gathered}
4 \mu \frac{m_{4}}{\eta}\left[\frac{m_{1}}{\eta}\left(Y_{2} \frac{m_{3}}{\eta} \frac{F_{3}}{\eta^{2}}-Y_{3} \frac{m_{2}}{\eta} \frac{F_{2}}{\eta^{2}}\right)-\frac{m_{2}}{\eta}\left(Y_{1} \frac{m_{3}}{\eta} \frac{F_{3}}{\eta^{2}}-Y_{3} \frac{m_{1}}{\eta} \frac{F_{1}}{\eta^{2}}\right)+\frac{m_{3}}{\eta}\left(Y_{1} \frac{m_{2}}{\eta} \frac{F_{2}}{\eta^{2}}-Y_{2} \frac{m_{1}}{\eta} \frac{F_{1}}{\eta^{2}}\right)\right] \\
-\left(\frac{m_{4}^{2}}{\eta^{2}}+1\right)\left[X_{1}\left(Y_{2} \frac{m_{3}}{\eta} \frac{F_{3}}{\eta^{2}}-Y_{3} \frac{m_{2}}{\eta} \frac{F_{2}}{\eta^{2}}\right)-X_{2}\left(Y_{1} \frac{m_{3}}{\eta} \frac{F_{3}}{\eta^{2}}-Y_{3} \frac{m_{1}}{\eta} \frac{F_{1}}{\eta^{2}}\right)+X_{3}\left(Y_{1} \frac{m_{2}}{\eta} \frac{F_{2}}{\eta^{2}}-Y_{2} \frac{m_{1}}{\eta} \frac{F_{1}}{\eta^{2}}\right)\right]=0
\end{gathered}
$$

where the expression for $X_{i}, Y_{i}, \frac{m_{i}}{\eta}, \frac{F_{i}}{\eta^{2}}$ and $\frac{F_{i}}{\eta^{2}}$ reduce accordingly. 


\section{Numerical Results and Discussion}

For computation of non-dimensional wave speed of Rayleigh wave, the following relevant physical constants for a thermoelastic solid half-space with diffusion and two-temperature are taken at $T_{0}=300 \mathrm{~K}$ $\lambda=5.775 \times 10^{10} \mathrm{~N} . \mathrm{m}^{-2}, \quad \mu=1.89 \times 10^{10} \mathrm{~N} . \mathrm{m}^{-2}, \quad \rho=2.7 \times 10^{3} \mathrm{Kg}^{-\mathrm{m}^{-3}}$, $c_{E}=2.361 \times 10^{2} J . K g^{-1} \cdot \mathrm{deg}^{-1}, K=0.494 \times 10^{2} W \cdot \mathrm{m}^{-1} . \mathrm{s}^{-1} \cdot \mathrm{deg}^{-1}$, $\tau_{0}=0.05 s, \quad a=0.005, \quad b=0.05$.

For above values of physical parameters, the equation (30) is solved by Fortran Program of iteration method to obtain the non-dimensional speed $c^{2} / c_{2}^{2}$ of Rayleigh wave for different ranges of thermo-diffusion parameter $D^{*}$, frequency $\chi$, two-temperature parameter $a^{*}$ and diffusion relaxation time $\tau$.

In Figure 1, the non-dimensional speed $c^{2} / c_{2}^{2}$ of Rayleigh wave is plotted against diffusion parameter $D^{*}$ varying from 0.2 to 1.0 , when $a^{*}=0.1,0.5$ and $0.9, \tau=0.04 s$ and $\chi=10 \mathrm{~Hz}$. For $a^{*}=0.5$, the value of non-dimensional speed is 1.0360 at $D^{*}=0.2$. It decreases with the increase in $D^{*}$ and attains its minimum value 0.3691 at $D^{*}=1$. This variation is shown by black curve in Figure 1 . The blue and red curves represent the variations for $a^{*}=0.1$ and $a^{*}=0.9$. The comparison of these three curves show the effect of two-temperature at different values of thermo-diffusion parameter $D^{*}$. The value of non-dimensional speed drops at each $D^{*}$ with the increase in value of two-temperature parameter $a^{*}$.

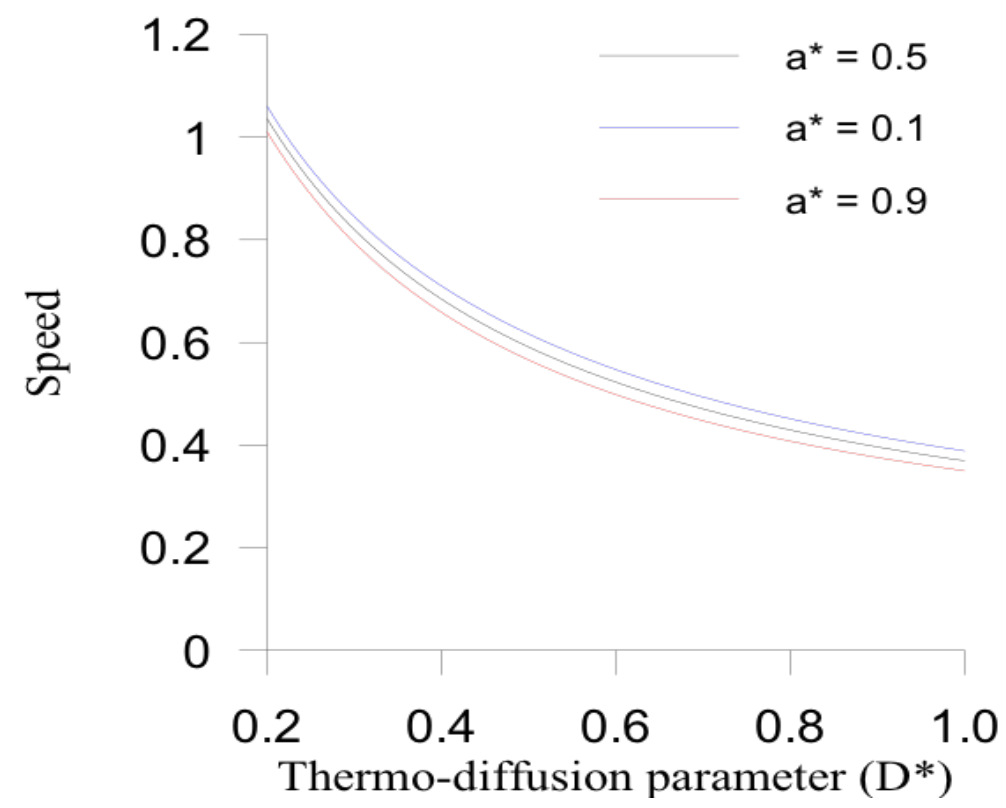

Figure 1. Variation of non-dimensional speed $\left(c^{2} / c_{2}^{2}\right)$ against thermodiffusion parameter $D^{*}$, when $a^{*}=0.1,0.5$ and 0.9 .

The non-dimensional speed $c^{2} / c_{2}^{2}$ of Rayleigh wave is plotted in Figure 2 against frequency $\chi$ varying from $1 \mathrm{~Hz}$ to $50 \mathrm{~Hz}$, when $a^{*}=0.1,0.5$ and $0.9, \tau=0.04 \mathrm{~s}$ and $D^{*}=0.5$. For $a^{*}=0.5$, the value of non-dimensional speed is 2.7754 at $\chi=1 \mathrm{~Hz}$. It decreases very sharply with the increase in $\chi$ and attains its minimum value 0.1232 at $\chi=50 \mathrm{~Hz}$. This variation is shown by black curve in Figure 2 . For $a^{*}=0.1$ and $a^{*}=0.9$, the variations of non-dimensional speed are shown by blue and red curves, respectively. The comparison of these three curves in Figure 2 show the effect of two-temperature at different values of frequency $\chi$.

In Figure 3, the non-dimensional speed $c^{2} / c_{2}^{2}$ of Rayleigh wave is plotted against two-temperature parameter $a^{*}$ from 0 to 1 , when $\chi=5,10$ and $50 \mathrm{~Hz}, D^{*}=0.5$ and $\tau=0.04 \mathrm{~s}$. For $\chi=10 \mathrm{~Hz}$, the value of non-dimensional speed is 0.6224 at $a^{*}=0$. It decreases slowly with the increase in $a^{*}$ and attains its minimum value 0.5594 at $a^{*}=1$. This variation is shown by black curve in Figure 3 . The blue and red curves in Figure 3 represent the variations for $\operatorname{ch} i=5$ and $\chi=50 \mathrm{~Hz}$. For $\chi=5 \mathrm{~Hz}$, the value of 


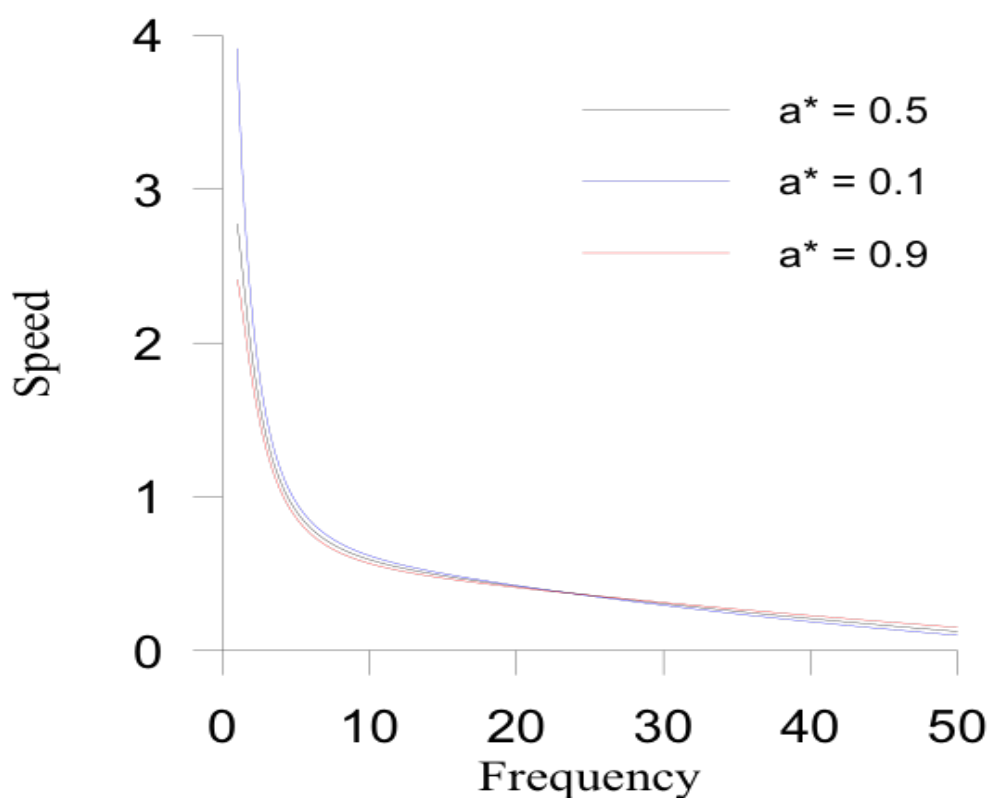

Figure 2. Variation of non-dimensional speed $\left(c^{2} / c_{2}^{2}\right)$ against frequency $\chi$, when $a^{*}=0.1,0.5$ and 0.9 .

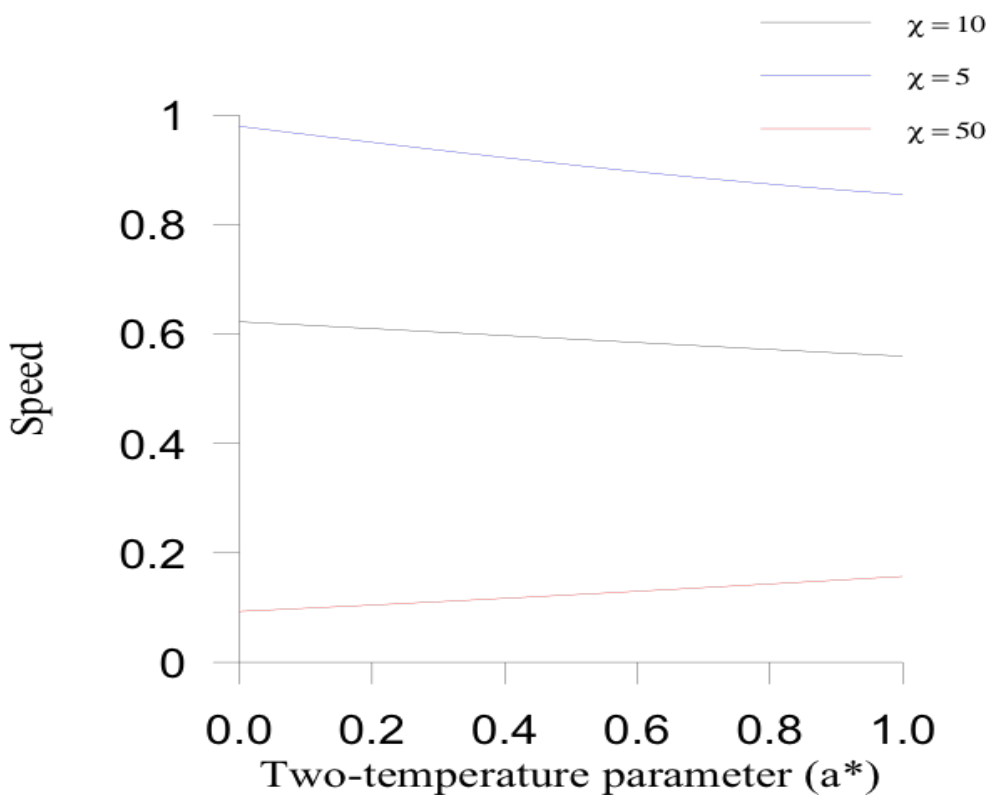

Figure 3. Variation of non-dimensional speed $\left(c^{2} / c_{2}^{2}\right)$ against two-temperature parameter $a^{*}$, when $\chi=5,10,50$.

non-dimensional speed is 0.9796 at $a^{*}=0$ and it decreases slowly with increase in value of $a^{*}$ and attains its minimum value 0.8549 at $a^{*}=1$. For $\chi=50 \mathrm{~Hz}$, the minimum value of non-dimensional speed is 0.0930 at $a^{*}=0$ and it increase slowly to its maximum value 0.1568 at $a^{*}=1$. The comparison of these three curves show the effect of frequency at different values of two-temperature parameter $a^{*}$.

The non-dimensional speed $c^{2} / c_{2}^{2}$ of Rayleigh wave is plotted in Figure 4 against diffusion relaxation time $\tau$ varying from 0 to $0.1 \mathrm{~s}$, when $a^{*}=0.1,0.5$ and $0.9, \chi=10 \mathrm{~Hz}$ and $D^{*}=0.5$. For $a^{*}=0.5$, the value of non-dimensional speed is 0.4796 at $\tau=0.1 \mathrm{~s}$. It increases sharply to its maximum value 0.7685 at $\tau=0.1 \mathrm{~s}$. This variation is shown by black curve in Figure 4 . For $a^{*}=0.1$ and $a^{*}=0.9$, the variations 


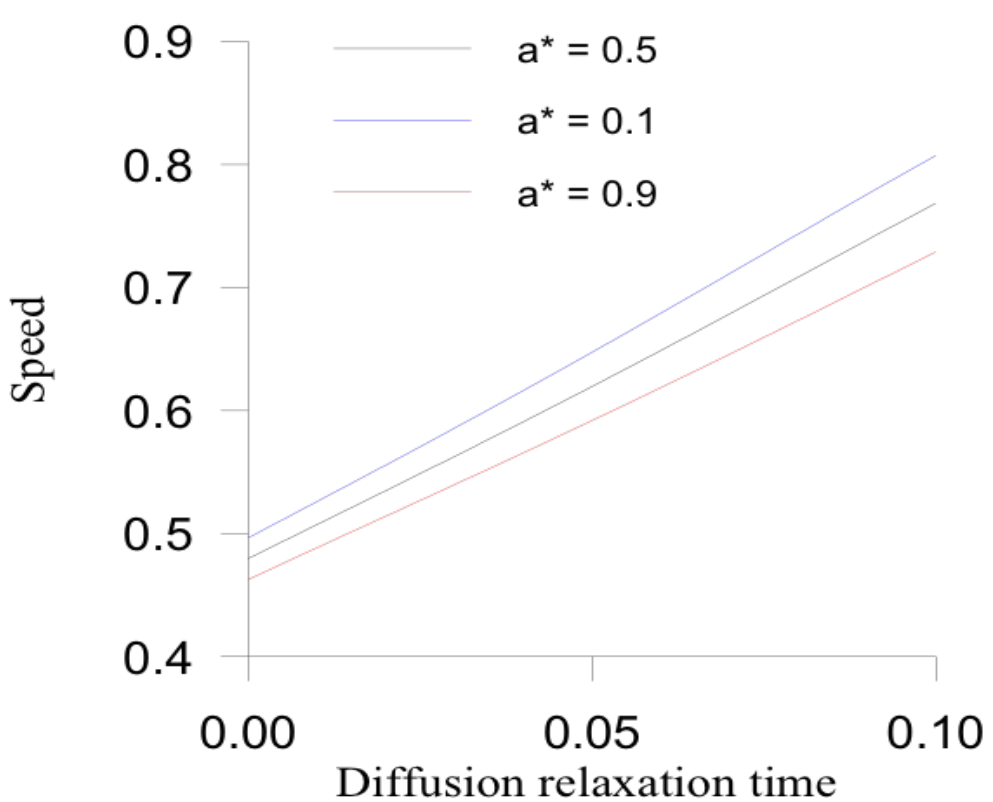

Figure 4. Variation of non-dimensional speed $\left(c^{2} / c_{2}^{2}\right)$ against diffusion relaxation time, when $a^{*}=0.1,0.5$ and 0.99 .

of non-dimensional speed are shown by blue and red curves, respectively. The comparison of these three curves in Figure 4 show the effect of two-temperature at different values of diffusion relaxation time $\tau$.

From above discussion, it is observed that the non-dimensional speed of Rayleigh wave depends on various material parameters. For lower frequency range, it decreases with increase in value of twotemperature parameter, whereas it increases in higher frequency range. The speed is much higher in low frequency range as compared to higher frequency range. The value of speed decreases with increase in value of thermo-diffusion parameter, whereas it increases with increase in value of diffusion relaxation time.

\section{Conclusion}

A secular equation for Rayleigh wave in a two-temperature thermoelastic medium with diffusion is obtained. For a given numerical values of the material constant, of the model, the wave speed of Rayleigh wave is computed and shown graphically. The theoretically as well as numerical results show the dependence of wave speed on thermal and diffusion parameters, two-temperature, relaxation times and frequency. Such type of theoretical problems may be helpful to experimental scientists working in the field of seismology, geophysics and non-destructive testing.

\section{References}

1. Biot, M. (1956) Thermoelasticity and irreversible thermo-dynamics, Journal of Applied Physics, 27, 249-253.

2. Chen, PJ and Gurtin, ME(1968) On a theory of heat conduction involving two temperatures, Zeitschrift fur angewandte Mathematik und Physik (ZAMP), 19, 614-627.

3. Chen, PJ and Gurtin, ME and Williams, WO (1968) A note on non simple heat conduction, Zeitschrift fur angewandte Mathematik und Physik (ZAMP), 19, 969-970.

4. Chen, PJ and Gurtin, ME and Williams, WO (1969) On the thermodynamics of non-simple elastic materials with two- temperatures, Zeitschrift fur angewandte Mathematik und Physik (ZAMP), 20, 107-112.

5. Dhaliwal, RS and Sherief, HH (1980) Generalized thermoelasticity for anisotropic media, Quarterly Applied Mathematics, 33, 1-8.

6. Green, AE and Lindsay, KA (1972) Thermoelasticity, Journal of Elasticity, 2, 1-7. 
7. Gurtin, ME and Williams, WO (1966) On the Clausius-Duhem inequality, Zeitschrift fur angewandte Mathematik und Physik (ZAMP), 17, 626-633.

8. Hetnarski, RB and Ignaczak, J (1999) Generalized Thermoelasticity, Journal of Thermal Stress, 22, 451-476.

9. Kumar R and Mukhopadhyay S (2010) Effects of thermal relaxation time on plane wave propagation under two-temperature thermoelasticity, International Journal of Engineering Science, 48, 128-139.

10. Lord H and Shulman Y (1967) A generalized dynamical theory of thermoelasticity, Journal of Mechanics and Physical Solids, 15, 299-309.

11. Nowacki W (1974) Dynamical problems of thermoelastic diffusion in solids I, II, Bull. Acad. Pol. Sci. Ser. Tech., 22, 55- 64, 129-135.

12. Nowacki W (1976) Dynamical problems of diffusion in solids, Engineering Fracture Mechanics, 8, 261-266.

13. Puri P and Jordan PM (2009) On the propagation of harmonic plane waves under the two-temperature theory, International Journal of Engineering Science, 44, 1113-1126.

14. Sherief HH and Saleh HA (2004) The theory of generalized thermoelastic difussion, International Journal of Engineering Science, 42, 591-608.

15. Singh B (2005) Reflection of P and SV waves from free surface of an elastic solid with generalized thermodiffusion, Journal of Earth System and Science, 114, 159-168.

16. Singh B (2006) Reflection of SV waves from free surface of an elastic solid in generalized thermoelastic diffusion, Journal of Sound and Vibration, 291, 764-778.

17. Singh B (2013) Propagation of Rayleigh wave in a two-temperature generalized thermoelastic solid half-space, ISRN Geophysics, 2013, Article ID 857937, 6 pages, doi:10.1155/2013/857937

18. Warren WE and Chen PJ (1973) Wave propagation in the two temperature theory of thermoelasticity, Acta Mechanica, 16, 21-23.

19. Youssef HM (2006) Theory of two-temperature generalized thermoelasticity, IMA Journal of Applied Mathematics, 71, 383-390.

\section{Appendix I}

The expressions for $L, M$ and $N$ in equation (18) are

$L=3 \eta^{2}-\left[\left(\bar{K}-a^{*}\right)\left(\chi^{2} \tau_{n}{ }^{*} c_{1}^{2}-D^{*} a \chi^{2}-\bar{\beta}_{2} D^{*} \beta_{2}\right)-D^{*} a\left(c_{1}{ }^{2}+\beta_{1}^{-} \epsilon_{1}\right)\right.$

$\left.+D^{*} b\left(\chi^{2} \epsilon_{2} a^{*}-\epsilon_{2} c_{1}^{2}\right)+D^{*} \beta_{2} \bar{\beta}_{2} a^{*}\right] /\left[a^{*} D^{*} b\left(c_{1}^{2} \epsilon_{2}+\bar{\beta}_{2} \epsilon_{1}\right)\right.$

$+\left(D^{*} a\left(a^{*} \epsilon_{1} \bar{\beta}_{1}-c_{1}^{2}\left(\bar{K}-a^{*}\right)\right)\right]$,

$M=3 \eta^{4}-2 \eta^{2}\left[\left(\bar{K}-a^{*}\right)\left(\chi^{2} \tau_{n}{ }^{*} c_{1}^{2}-D^{*} a \chi^{2}-\bar{\beta}_{2} D^{*} \beta_{2}\right)-D^{*} a\left(c_{1}^{2}+\beta_{1}^{-} \epsilon_{1}\right)\right.$

$+D^{*} b\left(\chi^{2} \epsilon_{2} a^{*}-\epsilon_{2} c_{1}^{2}\right)+D^{*} \beta_{2} \bar{\beta}_{2} a^{*}+\tau_{n}^{*}\left(\left(\bar{K}-a^{*}\right) \chi^{4}+\beta_{1} \epsilon_{1}+c_{1}^{2}\right)$

$\left.-\chi^{2}\left(D^{*} a+\epsilon_{2} D^{*} b\right)-D^{*} \beta_{2}\left(\bar{\beta}_{2}+\bar{\beta}_{1} \epsilon_{2}\right)\right] /\left[a^{*} D^{*} b\left(c_{1}^{2} \epsilon_{2}+\bar{\beta}_{2} \epsilon_{1}\right)\right.$

$+\left(D^{*} a\left(a^{*} \epsilon_{1} \bar{\beta}_{1}-c_{1}^{2}\left(\bar{K}-a^{*}\right)\right)\right]$,

$N=\eta^{6}-2 \eta^{4}\left[\left(\bar{K}-a^{*}\right)\left(\chi^{2} \tau_{n}{ }^{*} c_{1}{ }^{2}-D^{*} a \chi^{2}-\bar{\beta}_{2} D^{*} \beta_{2}\right)-D^{*} a\left(c_{1}{ }^{2}+\beta_{1}^{-} \epsilon_{1}\right)\right.$

$+D^{*} b\left(\chi^{2} \epsilon_{2} a^{*}-\epsilon_{2} c_{1}^{2}\right)+D^{*} \beta_{2} \bar{\beta}_{2} a^{*}+\tau_{n}^{*}\left(\left(\bar{K}-a^{*}\right) \chi^{4}+\beta_{1} \epsilon_{1}+c_{1}^{2}\right)$

$\left.-\chi^{2}\left(D^{*} a+\epsilon_{2} D^{*} b\right)-D^{*} \beta_{2}\left(\bar{\beta}_{2}+\bar{\beta}_{1} \epsilon_{2}\right)\right] /\left[a^{*} D^{*} b\left(c_{1}^{2} \epsilon_{2}+\bar{\beta}_{2} \epsilon_{1}\right)\right.$

$+\left(D^{*} a\left(a^{*} \epsilon_{1} \bar{\beta}_{1}-c_{1}^{2}\left(\bar{K}-a^{*}\right)\right)\right]$

$-\eta^{2}\left[\tau_{n}{ }^{*}\left(\left(\bar{K}-a^{*}\right) \chi^{4}+\beta_{1} \epsilon_{1}+c_{1}^{2}\right)-\chi^{2}\left(D^{*} a+\epsilon_{2} D^{*} b\right)\right.$

$\left.-D^{*} \beta_{2}\left(\bar{\beta}_{2}+\bar{\beta}_{1} \epsilon_{2}\right)\right] /\left[a^{*} D^{*} b\left(c_{1}^{2} \epsilon_{2}+\bar{\beta}_{2} \epsilon_{1}\right)+\left(D^{*} a\left(a^{*} \epsilon_{1} \bar{\beta}_{1}-c_{1}^{2}\left(\bar{K}-a^{*}\right)\right)\right]\right.$

$-\chi^{4} \tau_{n}{ }^{*} /\left[a^{*} D^{*} b\left(c_{1}{ }^{2} \epsilon_{2}+\bar{\beta}_{2} \epsilon_{1}\right)+\left(D^{*} a\left(a^{*} \epsilon_{1} \bar{\beta}_{1}-c_{1}{ }^{2}\left(\bar{K}-a^{*}\right)\right)\right]\right.$,

and, the expressions for $F_{i}$ and $F_{i}{ }^{*},(i=1,2,3)$ are

$$
\begin{aligned}
\frac{F_{i}}{\eta^{2}} & =\frac{\epsilon_{2}\left[c_{1}{ }^{2}\left(-1+\frac{m_{i}{ }^{2}}{\eta^{2}}\right)+\frac{\chi^{2}}{\eta^{2}}\right]+\bar{\beta}_{2} \epsilon_{1}\left(-1+\frac{m_{i}{ }^{2}}{\eta^{2}}\right)}{\epsilon_{2} \bar{\beta}_{1}\left[1-a^{*} \eta^{2}\left(-1+\frac{m_{i}{ }^{2}}{\eta^{2}}\right)\right]-\bar{\beta}_{2}\left[\bar{K} \eta^{2}\left(-1+\frac{m_{i}{ }^{2}}{\eta^{2}}\right)+\left(1-a^{*} \eta^{2}\left(-1+\frac{m_{i}{ }^{2}}{\eta^{2}}\right)\right)\right]}, \\
\frac{F_{i}^{*}}{\eta^{2}} & =\frac{D^{*} \beta_{2} \bar{\beta}_{1}\left(-1+\frac{m_{i}{ }^{2}}{\eta^{2}}\right)^{2}+D^{*} a\left(-1+\frac{m_{i}{ }^{2}}{\eta^{2}}\right)\left[c_{1}{ }^{2}\left(-1+\frac{m_{i}{ }^{2}}{\eta^{2}}\right)+\frac{\chi^{2}}{\eta^{2}}\right]}{\bar{\beta}_{1}\left[D^{*} b\left(-1+\frac{m_{i}{ }^{2}}{\eta^{2}}\right)+\frac{\chi^{2}}{\eta^{2}} \tau^{*}\right]+\bar{\beta}_{2} D^{*} a\left(-1+\frac{m_{i}{ }^{2}}{\eta^{2}}\right)} .
\end{aligned}
$$

